Research Article

\title{
Influence of Resistance Inducers on Nitrogen, Phosphorus and Potassium Contents of Susceptible Chickpea Cultivars after Inoculation with Ascochyta rabiei
}

\author{
Muhammad Usman Ghazanfar', Waqas Wakil' ${ }^{1}$, Shahbaz Talib Sahi ${ }^{3}$ and Waqas Raza ${ }^{1 *}$
}

${ }^{1}$ Department of Plant Pathology, College of Agriculture, University of Sargodha, 40100, Pakistan; ${ }^{2}$ Department of Agri. Entomology, University of Agriculture, Faisalabad 38000, Pakistan; ${ }^{3}$ Department of Plant Pathology, University of Agriculture, Faisalabad 38000, Pakistan.

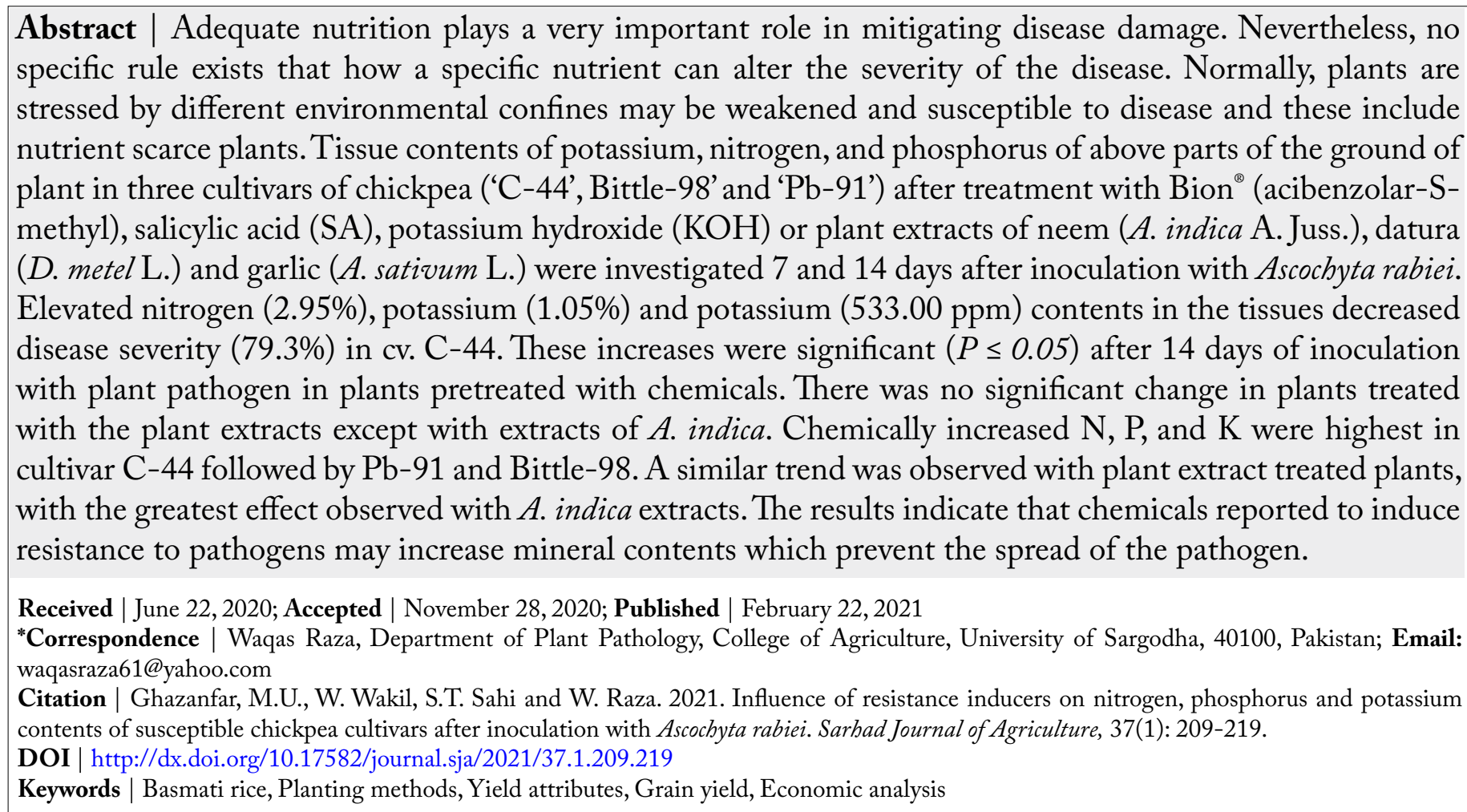

\section{Introduction}

Ohickpea (Cicer arietinum L.) is susceptible to different factors (biotic and a-biotic) that influence the production of the crop. Chickpea blight disease caused by the pathogen, Ascochyta rabiei (Pass) Labr, may result in up to $70 \%$ crop losses (Nene, 1984; Malik and Bashir, 1984) when environmental conditions are favorable for the disease. Although plant disease can be controlled by a range of cultural practices (Sattar, 1933; Luthra and Bedi, 1935) and chemo-therapentants (Tripathi et al., 1987; Singh and Singh, 1990), cultivation of resistant cultivars is mainly valuable and cost-effective practices (Nene and Reddy, 1987). Plant resistance to an infectious organism is governed by a lot of biochemical metabolites through which enzyme-catalyzed biochemical reactions (Tenhaken and Barz, 1991) depend on the accessibility of definite anion; cation, and enzymes (Chen and Strange, 1991). Nutrients 
play a very vital task in the development of plants and micro-organisms, so considered a significant factor for disease control strategies (Agrios, 2005). The severity of the disease is affected by all of the essential nutrients (Huber and Graham, 1999), and balanced nutrition necessary for any host plant to be resistant to pathogens (Filippi and Prabhu, 1998). The significance of plant nutrients in disease management strategy has been documented for many years but the accurate management of nutrients to manage some of the most severe diseases for sustainable agriculture have expected very slight attention (Huber and Graham, 1999).

Nitrogen is an important macro-nutrient obligatory for the normal development of plant parts (Chandra and Mishra, 1991). Nitrogen is an indispensable constituent of many metabolites like proteins, amino acids, phytochrome, nucleic acid, and chromosomes (Marschner, 1995). A deficiency of $\mathrm{N}$ results in impaired growth, reduction in protein synthesis, and inhibition of photosynthesis (Stitt and Krapp, 1998). Phosphorus is an integral part of many organic molecules such as cellular DNA, RNA, and ATP. It is also concerned with various metabolic processes in plants and pathogens but its task in resistance is unpredictable and not consistent (Kiraly, 1976). Potassium in plants has involved in various functions such as stomatal movement, enzyme activation, and turgor regulations (Peoples and Koch, 1979). Plants have deficient in potassium accumulate a considerable quantity of organic compounds because they function as an osmoticum in lack of adequate nutrients (potassium). High potassium status generally decreases the incidence of pests and disease $\%$ of the studies reported to decrease fungal and bacterial diseases due to a balanced $\mathrm{K}$ supply (Fuchs and Grossmann, 1972). The beneficial effect of potassium was instrumental in 69-70 (Amtmanns et al., 2009).

Plant leaf extracts of Azadirachta indica, Datura metal, and Allium satium have shown to be effective against Alternaria leaf spot, Rbizoctonia solani and Alternaria solani (Kagale et al., 2004; Guleria and Kumar, 2006; Slusarenko et al., 2008) by inducing resistance.

Minerals play a key role in different biochemical reactions concerned in resistance or susceptibility against various plant pathogens, study intended to conclude the influence of different treatments and inoculation with pathogen, $A$. rabiei, on the mineral content of chickpea cultivars differing in resistance to this pathogen.

\section{Materials and Methods}

\section{Climatic conditions}

The temperature range between 8 to $12^{\circ} \mathrm{C}$ during the night and 14 to $35^{\circ} \mathrm{C}$ through the day with $10-\mathrm{h}$ light period. The humidity ranges from $60-70 \%$.

\section{Plant material and experimental design}

Seeds of three chickpea cultivars (C-44, Bittle-98 and $\mathrm{Pb}-91)$ that are susceptible to $A$. rabiei were cultivated in small plots of $6.0 \mathrm{~m}^{2}$ in the field conditions. The experiment was a planned split plot design with varieties in the central plot, doses in subplots, and inducers in subplots with three replications. Each plot had six (6) rows and fifteen (15) chickpea plants per row.

\section{Preparation of plant extracts}

Plant extracts e.g, datura (Datura metel L.), garlic (Allium sativum L.), and neem (Azadirachta indica Juss.) were prepared from leaves collected from research areas from UAF, Pakistan while cloves of garlic were purchased from the local market. The leaves of selected plants were then washed in tap water, and rinsed with sterilized water before homogenizing in sterile distilled water $(1: 1 \mathrm{w} / \mathrm{v})$, and filtering through a muslin cloth. Fresh garlic extracts were prepared by removing the outer, dry peel and surface-sterilizing for $1 \mathrm{~min}$ in ethanol solution (70\%) and washed in sterile double distilled water. The cloves were firmed to a pulp and suspended in 100 $\mathrm{ml}$ distilled water and then filtered via muslin cloth. All extracts were heated at $40^{\circ} \mathrm{C}$ for almost ten (10) mint. to avoid contamination and diluted to different concentrations, 5,10 or $15 \%$ with double distilled water (v/v) (Jaganathan and Narasimhan, 1988).

\section{Resistance inducers chemicals}

Aqueous solutions $(0.5,1.0$, and $1.5 \mathrm{mM})$ of salicylic acid (Sigma Aldrich, Germany), Bion ${ }^{\oplus}$ (0.4, 0.8 and $1.2 \mathrm{mM}$, Syngenta Crop Protection, Germany) and 25,50 or $75 \mathrm{mM} \mathrm{KOH} \mathrm{(Sigma} \mathrm{Aldrich,} \mathrm{Germany)}$ were applied.

\section{Application of inducers and challenge inoculation with $A$. rabiei}

At early flowering, the chemicals and extract of plants were sprayed on plants until runoff while the check (control) plants were sprayed only with double 
distilled water. Pathogen inoculum was prepared and the conidial concentrations were adjusted with a hemocytometer (Ilyas and Khan, 1986). Four days after treatment, the plants were sprayed to run off with spore suspension of the plant pathogen, $A$. rabiei ( $1 \times 10^{5}$ spores $\left.\mathrm{L}^{-1}\right)$ that contained Tween 80 (3 drops/ liter) as a wetting agent in the late evening because the temperature was lesser at night to give better germination of spores (conidia). Spray inoculation continued for three days to guarantee maximum infection of plants. The plants were periodically sprayed with water $\left(\mathrm{H}_{2} \mathrm{O}\right)$ to maintain the moisture and favor germination of conidia.

\section{Processing of plant samples}

Shoot samples from treated inoculated and uninoculated chickpea plants of the three (3) cultivars were collected 7 and 14 days after inoculation at which time symptoms were fully developed on the inoculated control plants. The shoots were washed with a detergent solution $(0.2 \%)$ to remove dirt, washed in $\mathrm{HCl}$ $(0.8 \%)$ to get rid of metallic contaminants, and then washed in deionized water to eliminate the prior two solutions. The samples were dried up on paper towels in the shade and placed in paper bags before drying in the oven for $72 \mathrm{~h}$ at $70^{\circ} \mathrm{C}$ to get constant weight. These samples were ground with the grinder and then analyzed Nitrogen, Phosphorus, and potassium (Bhargava and Raghupathi, 1995; Karla and Maynard, 1991). Nitrogen and Phosphorus were recorded as a percentage of dry weight and potassium as ppm.

\section{Statistical analysis}

Experimental data were evaluated by ANOVA and the means were separated by Tukey's HSD test at $5 \%$ level of significance (Steel et al., 1997) using the software R. 2.12.1 (2008). The experiment was repeated three times independently to overcome the pseudo replications.

\section{Results and Discussion}

In a preliminary experiment, resistance was induced in three chickpea cultivars by the application of Bion ${ }^{\oplus}$ (acibenzolar-S-methyl), salicylic acid (SA), potassium hydroxide $(\mathrm{KOH})$, and plant extracts of garlic, neem, and datura. The data recorded considerable disease fall $(79.3 \%)$ was observed by Bion ${ }^{\circledR}$ in the cv. C-44 (@1.2 mM (Figure 1B) compared to SA (salicylic acid), however, the least was observed by potassium hydro-oxide. Among different plant extracts tested, the utmost disease reduction (43.5\%) against the disease was seen after application of $A$. indica (Figure 1D) and other extracts, D. metel and $A$. sativum did not attest effective to reduce the disease.

\section{Nitrogen}

Salicylic acid significantly increased $(P \leq 0.05)$ nitrogen in inoculated plants (Table 1). This increase was highest in cultivar C-44 (2.95\%) intermediate in $\mathrm{Pb}-91$ (2.65 \% and the least in Bittle-98 (2.51\%). In all the three cultivars, the increase in nitrogen was highest with the highest rate of salicylic acid and inoculation with the pathogen. Bion (with $1.2 \mathrm{mM}$ ) increased $\mathrm{N}$ to $2.86 \%$ in $\mathrm{C}-44,2.76 \%$ in $\mathrm{Pb}-91$ and $2.48 \%$ in Bittle-98 14 days after treatment in inoculated plants. $\mathrm{KOH}$ increased nitrogen in the three cultivars the least $(1.48,1.3$, and $1.26 \%$ in $\mathrm{C}-44, \mathrm{~Pb}-91$, and Bittle-98, respectively) (Table 1). Plant extracts did not significantly increase nitrogen. Overall, the application of chemicals increased nitrogen more than the plant extracts with the increase more pronounced by salicylic acid at the highest rate tested and the least with by $\mathrm{KOH}$ in cultivar $\mathrm{C}-44$.

\section{Phosphorus}

The phosphorus contents (Table 2) of chemically treated inoculated and un-inoculated plants of three chickpea cultivars were notably different $(P \leq 0.05)$. Generally, the phosphorus in all three inoculated cultivars was increased 14 days after treatment by the higher rate of chemicals and plant extracts except with $\mathrm{KOH}$ where $\mathrm{P}$ in the treated, un-inoculated plants was higher $(0.51,0.63$, and $0.78 \%)$ compared to treated inoculated plants $(0.35,0.45$ and 0.49 percent) of cultivar C-44 but opposite in $\mathrm{Pb}-91$ and Bittle-98. The plant extracts did not appreciably change the amount of phosphorus in the three chickpea cultivars.

\section{Potassium}

Potassium in the chemically treated, inoculated C-44 cultivar ranged from 480.8 to $527.86 \mathrm{ppm}$ and was significantly higher than salicylic acid treated un-inoculated plants (Table 3). Cultivars $\mathrm{Pb}-91$ and Bittle- 98 also showed the same trend with a maximum of $310.1 \mathrm{ppm}$ and $158.3 \mathrm{ppm}$ potassium 14 days after treatment and inoculation. The highest $\mathrm{K}$ (533.0 ppm) was with the highest rate of Bion, The potassium in $\mathrm{Pb}-91$ and Bittle-98 was also increased by Bion and inoculation. The lowest potassium (79.16 ppm) was in Bittle-98 which was significantly lower than $\mathrm{Pb}-91$ and $\mathrm{C}-44$ after application of $\mathrm{KOH}$ and 

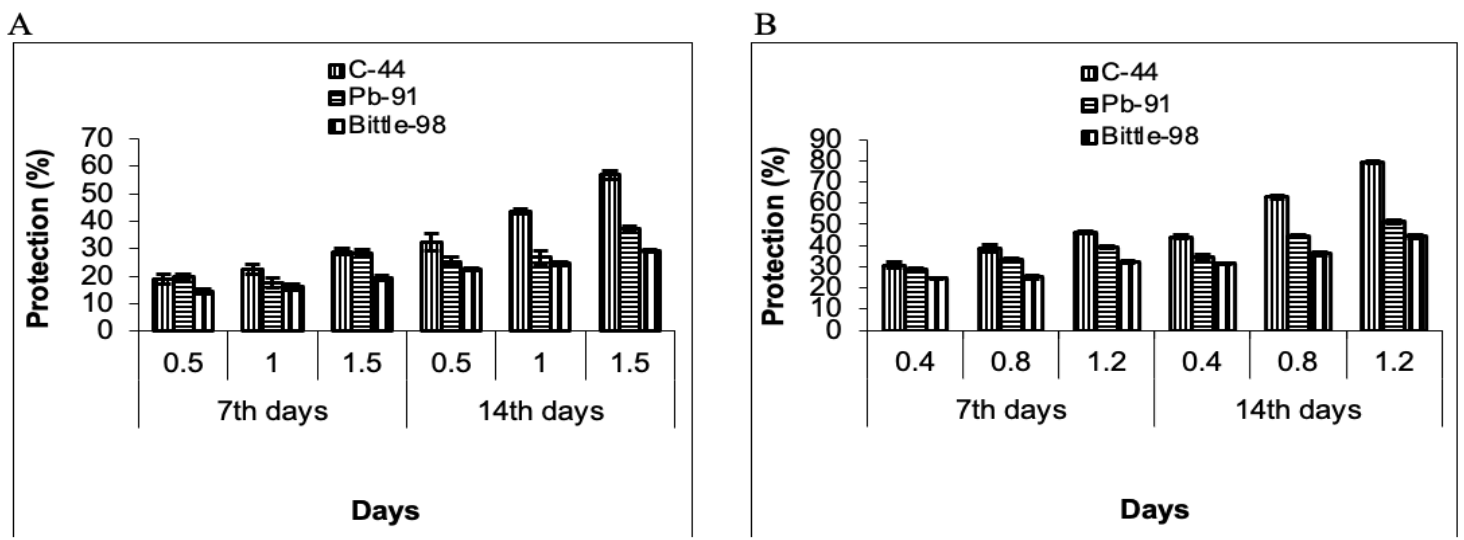

C

$\mathrm{D}$
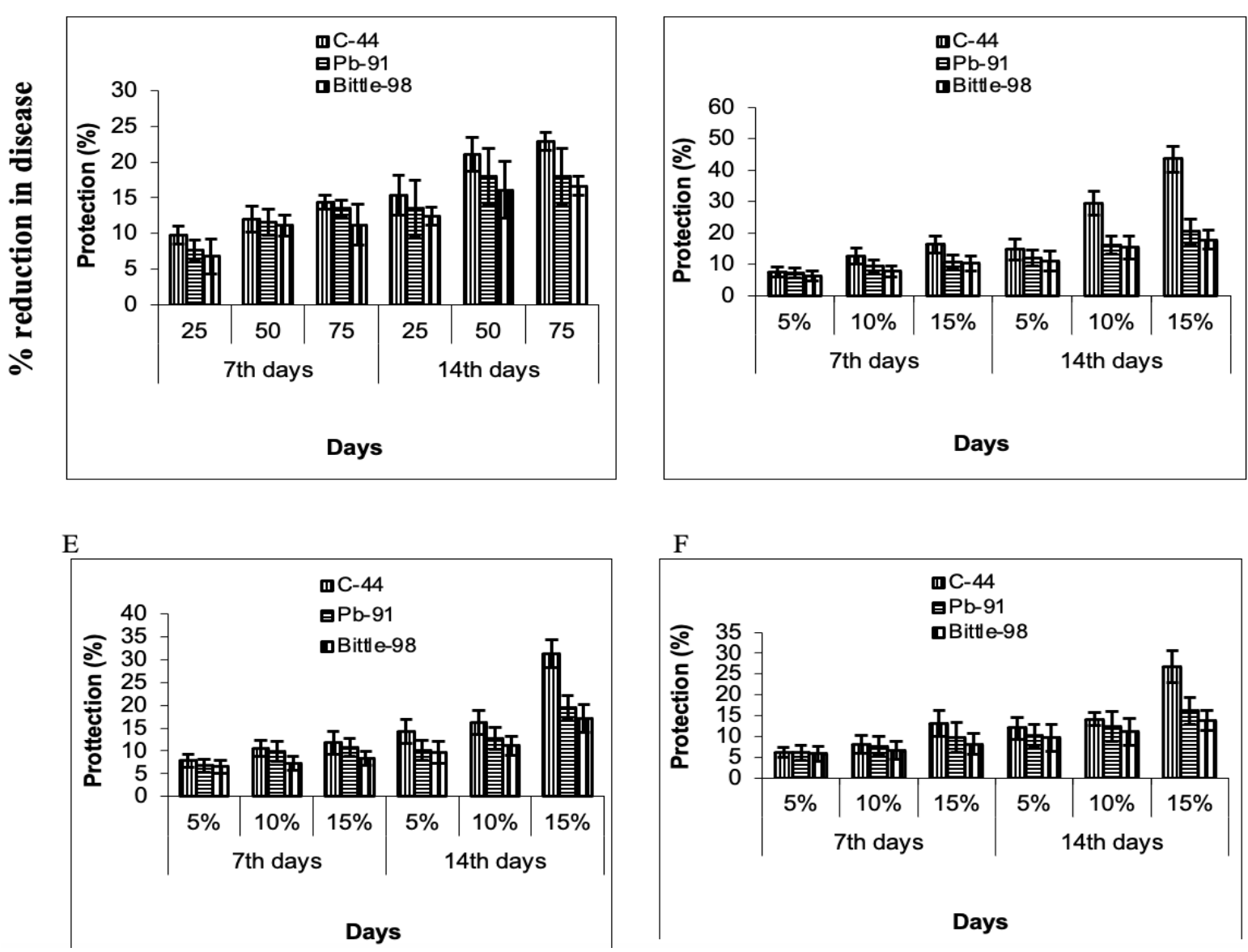

Figure 1: Mean comparison of \% reduction in Ascochyta blight disease by the application of $(A)$ salicylic acid $(B)$ Bion $(C) K O H(D) N e e m$ (E) Dautra $(F)$ Garlic in three chickpea cultivars. Data are means of three repeated experiments. The error bar represents the standard error of the mean according to the least significant difference test at $P \leq 0.05$.

inoculation with $A$. rabiei. Increased Potassium also was observed in chemically treated un-inoculated plants. Plant extracts also increased potassium in plant tissues but too much lower levels compared to the chemicals. The potassium in C-44 (174.89 ppm) was highest 14 days after treatment with neem extract compared with the treated un-inoculated C-44 plant (69.4 ppm) (Table 3). The lowest potassium (29.2 ppm) was found in Bittle-98 treated with the highest rate of garlic extract.

The nutritional status and availability influence the development and metabolisms of the plant as well as their level of susceptibility or resistance to pathogens (Krauss, 1999; Ruan and Wu, 2000). Successful invasion of pathogens not only depends upon their ability to evade defense mechanisms but also to utilize the nutrients available in plants (Solomon $e t$ al., 2003). In the current study, we investigated the impact of several chemicals reported to induce disease resistance on nitrogen, phosphorus, and potassium content after inoculation with $A$. rabiei in three chickpea cultivars. We found that nitrogen increased and that was more pronounced after inoculation with 
A. rabiei. Although the varieties under study were susceptible to Ascochyta blight yet by the application of resistance inducers they showed a reduction in the disease with increase nitrogen contents (Ghazanfar et al., 2010). The reasons for this increase might be due to fungal autolysis, increased nitrogen assimilation, increased flux from intercellular sources, and increased apoplast protease activity (Soloman and Oliver, 2001). In earlier studies, the effect of nitrogen on disease progress was variable but the causes of this irregularity are inadequately understood (Hoffland et al., 2000). The results presented here are consistent with studies of Randhawa (1994) who reported an increase of nitrogen in chickpea cultivars after inoculation with A. rabiei. Comparable results also have been reported by Soloman and Oliver (2001) in describing higher total nitrogen in tomato leaves after inoculation with Cladosporium flavum. Nitrogen increased in lentil plants after inoculation with $A$. lentis and this increase was higher in the susceptible clusters compare with the resistant ones as described by Sahi et al. (2007). The supply of nitrogen greatly influences the activity of three resistance-related enzymes: Chitinases, chitosanases, and preoxidases, and their level were much lower in plants cultivated under limited Nitrogen than high nitrogen conditions (Dietrich et al., 2004).

Table 1: Mean comparison ( \pm S.E) of nitrogen content (\% dry weight) in cultivars $\mathrm{C}-44, \mathrm{~Pb}-91$ and Bittle-98 after 7 and 14 days of the treatments with resistance inducers (chemicals and plant extracts).

\begin{tabular}{|c|c|c|c|c|c|c|c|c|c|c|}
\hline \multirow{3}{*}{\multicolumn{2}{|c|}{ Treatments }} & \multicolumn{9}{|l|}{ Cultivars } \\
\hline & & C-44 & & & $\mathrm{Pb}-91$ & & & Bittle-98 & & \\
\hline & & \multicolumn{9}{|l|}{ Doses (Mm) } \\
\hline \multicolumn{2}{|c|}{ Salicylic acid } & 0.5 & 1.0 & 1.5 & 0.5 & 1.0 & 1.5 & 0.5 & 1.0 & 1.5 \\
\hline \multirow[t]{2}{*}{$7 d$} & Ind. and Un- & $1.64 \pm 0.022$ & $1.88 \pm 0.008$ & $1.97 \pm 0.008$ & $1.42 \pm 0.017$ & $1.46 \pm 0.013$ & $1.56 \pm 0.021$ & $1.14 \pm 0.025$ & $1.21 \pm 0.017$ & $1.47 \pm 0.008$ \\
\hline & Ind. and Inoc & $2.33 \pm 0.007$ & $2.55 \pm 0.030$ & $2.77 \pm 0.021$ & $2.30 \pm 0.053$ & $2.40 \pm 0.056$ & $2.61 \pm 0.008$ & $2.18 \pm 0.005$ & $2.30 \pm 0.054$ & $2.46 \pm 0.006$ \\
\hline \multirow[t]{2}{*}{$14 d$} & Ind. and Un-Inoc. & $1.78 \pm 0.013$ & $1.93 \pm 0.025$ & $1.99 \pm 0.064$ & $1.49 \pm 0.005$ & $1.51 \pm 0.007$ & $1.62 \pm 0.051$ & $1.40 \pm 0.004$ & $1.45 \pm 0.011$ & $1.51 \pm 0.003$ \\
\hline & Ind. and Inoc & $2.37 \pm 0.053$ & $2.59 \pm 0.055$ & $2.95 \pm 0.027$ & $2.33 \pm 0.038$ & $2.46 \pm 0.050$ & $2.65 \pm 0.027$ & $2.31 \pm 0.016$ & $2.36 \pm 0.012$ & $2.51 \pm 0.006$ \\
\hline \multicolumn{2}{|c|}{ Bion } & 0.4 & 0.8 & 1.2 & 0.4 & 0.8 & 1.2 & 0.4 & 0.8 & .2 \\
\hline \multirow[t]{2}{*}{$7 d$} & Ind. and Un & $2.22 \pm 0.013$ & $2.22 \pm 0.008$ & $2.32 \pm 0.015$ & $1.51 \pm 0.011$ & $1.63 \pm 0.02$ & $1.71 \pm 0.007$ & $1.40 \pm 0.023$ & $1.49 \pm 0.024$ & $1.53 \pm 0.016$ \\
\hline & Ind. an & $2.67 \pm 0.011$ & $2.70 \pm 0.023$ & $2.75 \pm 0.026$ & $2.46 \pm 0.016$ & $2.65 \pm 0.03$ & $2.71 \pm 0.009$ & $2.13 \pm 0.031$ & $2.25 \pm 0.020$ & $2.45 \pm 0.019$ \\
\hline \multirow[t]{2}{*}{$14 d$} & Ind. and Un-Inoc. & $2.22 \pm 0.014$ & $2.35 \pm 0.017$ & $2.37 \pm 0.018$ & $1.57 \pm 0.013$ & $1.70 \pm 0.02$ & $1.76 \pm 0.011$ & $1.49 \pm 0.019$ & $1.51 \pm 0.017$ & $1.60 \pm 0.027$ \\
\hline & Ind. and Inoc & $2.68 \pm 0.010$ & $2.81 \pm 0.012$ & $2.86 \pm 0.013$ & $2.54 \pm 0.027$ & $2.68 \pm 0.01$ & $2.76 \pm 0.013$ & $2.31 \pm 0.041$ & $2.36 \pm 0.013$ & $2.48 \pm 0.061$ \\
\hline \multicolumn{2}{|c|}{$\mathrm{KOH}$} & 25 & 50 & 75 & 25 & 50 & 75 & 25 & 50 & 75 \\
\hline \multirow[t]{2}{*}{$7 d$} & Ind. and Un-Inoc. & $0.82 \pm 0.006$ & $0.94 \pm 0.016$ & $0.99 \pm 0.009$ & $0.71 \pm 0.012$ & $0.73 \pm 0.014$ & $0.78 \pm 0.006$ & $0.57 \pm 0.010$ & $0.61 \pm 0.024$ & $0.74 \pm 0.015$ \\
\hline & Ind. and Inoc & $1.17 \pm 0.004$ & $1.28 \pm 0.012$ & $1.39 \pm 0.014$ & $1.15 \pm 0.031$ & $1.20 \pm 0.047$ & $1.31 \pm 0.013$ & $1.09 \pm 0.011$ & $1.15 \pm 0.008$ & $1.23 \pm 0.019$ \\
\hline \multirow[t]{2}{*}{$14 d$} & Ind. and U & $0.89 \pm 0.011$ & $0.97 \pm 0.010$ & $1.00 \pm 0.021$ & $0.75 \pm 0.023$ & $0.76 \pm 0.007$ & $0.81 \pm 0.008$ & $0.70 \pm 0.021$ & $0.73 \pm 0.011$ & $0.76 \pm 0.013$ \\
\hline & Ind. an & $1.19 \pm 0.013$ & $1.30 \pm 0.027$ & $1.48 \pm 0.017$ & $1.17 \pm 0.014$ & $1.23 \pm 0.016$ & $1.33 \pm 0.011$ & $1.16 \pm 0.013$ & $1.18 \pm 0.013$ & $1.26 \pm 0.017$ \\
\hline \multicolumn{2}{|c|}{ A. indica } & $5 \%$ & $10 \%$ & $15 \%$ & $5 \%$ & $10 \%$ & $15 \%$ & $5 \%$ & $10 \%$ & - \\
\hline \multirow[t]{2}{*}{$7 \mathrm{~d}$} & Ind. an & $0.53 \pm 0.01$ & $0.61 \pm 0.005$ & $0.64 \pm 0.013$ & $0.46 \pm 0.011$ & $0.47 \pm 0.006$ & $0.51 \pm 0.009$ & $0.37 \pm 0.010$ & $0.40 \pm 0.004$ & $0.48 \pm 0.011$ \\
\hline & Ind. ar & $0.76 \pm 0.01$ & $0.82 \pm 0.016$ & $0.91 \pm 0.021$ & $0.74 \pm 0.017$ & $0.78 \pm 0.008$ & $0.86 \pm 0.021$ & $0.71 \pm 0.014$ & $0.75 \pm 0.017$ & $0.80 \pm 0.018$ \\
\hline \multirow[t]{2}{*}{$14 \mathrm{~d}$} & Ind. and Un-Inoc. & $0.57 \pm 0.02$ & $0.63 \pm 0.014$ & $0.66 \pm 0.014$ & $0.49 \pm 0.019$ & $0.50 \pm 0.011$ & $0.53 \pm 0.013$ & $0.46 \pm 0.011$ & $0.47 \pm 0.008$ & $0.49 \pm 0.009$ \\
\hline & Ind. and Inoc & $0.78 \pm 0.01$ & $0.84 \pm 0.017$ & $0.96 \pm 0.011$ & $0.76 \pm 0.012$ & $0.81 \pm 0.016$ & $0.88 \pm 0.017$ & $0.76 \pm 0.013$ & $0.78 \pm 0.013$ & $0.82 \pm 0.014$ \\
\hline \multicolumn{2}{|c|}{ D. metel } & $5 \%$ & $10 \%$ & $15 \%$ & $5 \%$ & $10 \%$ & $15 \%$ & $5 \%$ & $10 \%$ & $15 \%$ \\
\hline \multirow[t]{2}{*}{$7 d$} & Ind. and Un-Inoc. & $0.34 \pm 0.002$ & $0.39 \pm 0.011$ & $0.41 \pm 0.005$ & $0.30 \pm 0.004$ & $0.30 \pm 0.011$ & $0.33 \pm 0.016$ & $0.23 \pm 0.007$ & $0.26 \pm 0.004$ & $0.32 \pm 0.012$ \\
\hline & Ind. and Inoc & $0.48 \pm 0.008$ & $0.54 \pm 0.010$ & $0.59 \pm 0.011$ & $0.48 \pm 0.009$ & $0.51 \pm 0.017$ & $0.55 \pm 0.006$ & $0.45 \pm 0.011$ & $0.48 \pm 0.011$ & $0.52 \pm 0.017$ \\
\hline \multirow[t]{2}{*}{$14 \mathrm{~d}$} & Ind. ar & $0.37 \pm 0.011$ & $0.40 \pm 0.008$ & $0.42 \pm 0.010$ & $0.30 \pm 0.012$ & $0.32 \pm 0.014$ & $0.34 \pm 0.005$ & $0.29 \pm 0.005$ & $0.31 \pm 0.008$ & $0.33 \pm 0.009$ \\
\hline & Ind. anc & $0.50 \pm 0.013$ & $0.57 \pm 0.006$ & $0.64 \pm 0.002$ & $0.50 \pm 0.005$ & $0.53 \pm 0.008$ & $0.56 \pm 0.015$ & $0.49 \pm 0.003$ & $0.50 \pm 0.017$ & $0.53 \pm 0.021$ \\
\hline \multicolumn{2}{|c|}{ A. sativum } & $5 \%$ & $10 \%$ & $15 \%$ & $5 \%$ & $10 \%$ & $15 \%$ & $5 \%$ & $10 \%$ & $15 \%$ \\
\hline \multirow[t]{2}{*}{$7 \mathrm{~d}$} & Ind. and Un-Inoc. & $0.26 \pm 0.006$ & $0.30 \pm 0.011$ & $0.32 \pm 0.007$ & $0.21 \pm 0.010$ & $0.24 \pm 0.013$ & $0.26 \pm 0.011$ & $0.17 \pm 0.004$ & $0.20 \pm 0.010$ & $0.24 \pm 0.012$ \\
\hline & Ind. and Inoc & $0.39 \pm 0.004$ & $0.43 \pm 0.008$ & $0.47 \pm 0.015$ & $0.38 \pm 0.012$ & $0.39 \pm 0.008$ & $0.43 \pm 0.013$ & $0.34 \pm 0.011$ & $0.38 \pm 0.013$ & $0.40 \pm 0.013$ \\
\hline \multirow[t]{2}{*}{$14 \mathrm{~d}$} & Ind. and Un-In & $0.28 \pm 0.010$ & $0.33 \pm 0.005$ & $0.34 \pm 0.019$ & $0.23 \pm 0.006$ & $0.26 \pm 0.021$ & $0.27 \pm 0.009$ & $0.24 \pm 0.013$ & $0.25 \pm 0.011$ & $0.25 \pm 0.010$ \\
\hline & Ind. and Inoc & $0.40 \pm 0.012$ & $0.44 \pm 0.010$ & $0.50 \pm 0.016$ & $0.38 \pm 0.014$ & $0.45 \pm 0.018$ & $0.48 \pm 0.014$ & $0.38 \pm 0.014$ & $0.39 \pm 0.012$ & $0.43 \pm 0.021$ \\
\hline
\end{tabular}

$H S D$ value for treatments $=0.02912 ; H S D$ value for doses $=0.00090 ; H S D$ value for varieties $=0.01494$. 
Table 2: Mean comparison ( \pm S.E) of phosphorus content (\% dry weight) in cultivars $C-44, P b-91$ and Bittle-98 after 7 and 14 days of the treatments with resistance inducers (chemicals and plant extracts).

\begin{tabular}{|c|c|c|c|c|c|c|c|c|c|c|}
\hline \multirow{3}{*}{\multicolumn{2}{|c|}{ Treatments }} & \multicolumn{9}{|l|}{ Cultivars } \\
\hline & & $\mathrm{C}-44$ & & & $\mathrm{~Pb}-91$ & & & Bittle-98 & & \\
\hline & & \multicolumn{9}{|l|}{ Doses (Mm) } \\
\hline \multicolumn{2}{|c|}{ Salicylic acid } & 0.5 & 1.0 & 1.5 & 0.5 & 1.0 & 1.5 & 0.5 & 1.0 & 1.5 \\
\hline \multirow[t]{2}{*}{$7 d$} & Ind. and Un-Inoc. & $0.40 \pm 0.008$ & $0.58 \pm 0.011$ & $0.73 \pm 0.019$ & $0.33 \pm 0.006$ & $0.44 \pm 0.012$ & $0.54 \pm 0.006$ & $0.16 \pm 0.002$ & $0.25 \pm 0.004$ & $0.34 \pm 0.010$ \\
\hline & Ind. and Inoc & $0.68 \pm 0.023$ & $0.88 \pm 0.012$ & $0.95 \pm 0.014$ & $0.43 \pm 0.011$ & $0.54 \pm 0.014$ & $0.68 \pm 0.012$ & $0.26 \pm 0.001$ & $0.33 \pm 0.007$ & $0.45 \pm 0.011$ \\
\hline \multirow[t]{2}{*}{$14 d$} & Ind. and Un-Inoc. & $0.51 \pm 0.013$ & $0.63 \pm 0.014$ & $0.78 \pm 0.011$ & $0.38 \pm 0.012$ & $0.47 \pm 0.004$ & $0.62 \pm 0.019$ & $0.18 \pm 0.011$ & $0.26 \pm 0.011$ & $0.40 \pm 0.009$ \\
\hline & Ind. and Inoc & $.70 \pm 0.020$ & $0.91 \pm 0.019$ & $0.98 \pm 0.016$ & $0.47 \pm 0.014$ & $0.56 \pm 0.010$ & $0.72 \pm 0.020$ & $0.30 \pm 0.016$ & $0.42 \pm 0.013$ & $0.52 \pm 0.016$ \\
\hline \multicolumn{2}{|c|}{ Bion } & 0.4 & 0.8 & 1.2 & 0.4 & 0.8 & 1.2 & 0.4 & 0.8 & 1.2 \\
\hline \multirow[t]{2}{*}{$7 d$} & Ind. an & $0.54 \pm 0.011$ & $0.65 \pm 0.013$ & $0.73 \pm 0.018$ & $0.38 \pm 0.003$ & $0.49 \pm 0.002$ & $0.57 \pm 0.010$ & $0.18 \pm 0.002$ & $0.28 \pm 0.006$ & $0.40 \pm 0.010$ \\
\hline & Ind. and Inoc & $0.83 \pm 0.013$ & $0.91 \pm 0.027$ & $0.98 \pm 0.006$ & $0.48 \pm 0.010$ & $0.58 \pm 0.005$ & $0.72 \pm 0.011$ & $0.30 \pm 0.006$ & $0.40 \pm 0.003$ & $0.52 \pm 0.013$ \\
\hline \multirow[t]{2}{*}{$14 d$} & Ind. and Un-Inoc. & $0.62 \pm 0.005$ & $0.69 \pm 0.023$ & $0.76 \pm 0.011$ & $0.41 \pm 0.004$ & $0.52 \pm 0.003$ & $0.60 \pm 0.020$ & $0.22 \pm 0.003$ & $0.32 \pm$ & 0.005 \\
\hline & Ind. and Inoc & $0.83 \pm 0.024$ & $0.96 \pm 0.020$ & $1.05 \pm 0.023$ & $0.51 \pm 0.011$ & $0.61 \pm 0.011$ & $0.79 \pm 0.014$ & $0.39 \pm 0.010$ & 0.46 & $0.59 \pm 0.011$ \\
\hline \multicolumn{2}{|c|}{$\mathrm{KOH}$} & 25 & 50 & 75 & 25 & 50 & 75 & 25 & 50 & 75 \\
\hline \multirow[t]{2}{*}{$7 \mathrm{~d}$} & Ind. and Un- & $0.20 \pm 0.003$ & $0.29 \pm 0.004$ & $0.36 \pm 0.009$ & $0.17 \pm 0.004$ & $0.22 \pm 0.006$ & $0.27 \pm 0.020$ & $0.08 \pm 0.001$ & $0.13 \pm 0.002$ & 0.17 \\
\hline & Ind. and Inoc & $0.34 \pm 0.016$ & $0.44 \pm 0.019$ & $0.48 \pm 0.010$ & $0.22 \pm 0.003$ & $0.27 \pm 0.001$ & $0.34 \pm 0.011$ & $0.13 \pm 0.001$ & $0.17 \pm 0.010$ & $0.22 \pm 0.005$ \\
\hline \multirow[t]{2}{*}{$14 d$} & Ind. and & $0.51 \pm 0.021$ & $0.63 \pm 0.023$ & $0.78 \pm 0.013$ & $0.19 \pm 0.001$ & $0.24 \pm 0.002$ & $0.31 \pm 0.017$ & $0.09 \pm 0.002$ & $0.13 \pm 0.003$ & $0.20 \pm 0.011$ \\
\hline & Ind. and Inoc & $0.35 \pm 0.006$ & $0.45 \pm 0.009$ & $0.49 \pm 0.017$ & $0.23 \pm 0.011$ & $0.28 \pm 0.003$ & $0.36 \pm 0.013$ & $0.15 \pm 0.004$ & $0.21 \pm 0.001$ & $0.26 \pm 0.017$ \\
\hline \multicolumn{2}{|c|}{ A. indica } & $5 \%$ & $10 \%$ & $15 \%$ & $5 \%$ & $10 \%$ & $15 \%$ & $5 \%$ & $10 \%$ & $15 \%$ \\
\hline \multirow[t]{2}{*}{$7 d$} & Ind. and Un-Inoc. & $0.12 \pm 0.002$ & $0.19 \pm 0.001$ & $0.24 \pm 0.010$ & $0.11 \pm 0.003$ & $0.14 \pm 0.006$ & $0.18 \pm 0.010$ & $0.05 \pm 0.001$ & $0.08 \pm 0.001$ & $0.11 \pm 0.002$ \\
\hline & Ind. and Inoc & $0.23 \pm 0.011$ & $0.29 \pm 0.006$ & $0.32 \pm 0.009$ & $0.13 \pm 0.006$ & $0.18 \pm 0.003$ & $0.22 \pm 0.003$ & $0.09 \pm 0.010$ & $0.11 \pm 0.003$ & $0.15 \pm 0.003$ \\
\hline \multirow[t]{2}{*}{$14 d$} & Ind. and Un-Inoc. & $0.33 \pm 0.003$ & $0.41 \pm 0.020$ & $0.51 \pm 0.013$ & $0.12 \pm 0.010$ & $0.15 \pm 0.014$ & $0.20 \pm 0.006$ & $0.06 \pm 0.001$ & $0.09 \pm 0.001$ & $0.13 \pm 0.010$ \\
\hline & Ind. and Inoc & $0.25 \pm 0.012$ & $0.31 \pm 0.016$ & $0.35 \pm 0.011$ & $0.15 \pm 0.011$ & $0.19 \pm 0.010$ & $0.24 \pm 0.007$ & $0.11 \pm 0.003$ & $0.14 \pm 0.004$ & $0.17 \pm 0.006$ \\
\hline \multicolumn{2}{|c|}{ D. metel } & $5 \%$ & $10 \%$ & $15 \%$ & $5 \%$ & $10 \%$ & $15 \%$ & $5 \%$ & $10 \%$ & $15 \%$ \\
\hline \multirow[t]{2}{*}{$7 \mathrm{~d}$} & Ind. and Un-In & $0.08 \pm 0.001$ & $0.12 \pm 0.010$ & $0.16 \pm 0.003$ & $0.07 \pm 0.001$ & $0.09 \pm 0.002$ & $0.11 \pm 0.010$ & $0.04 \pm 0.001$ & $0.06 \pm 0.001$ & $0.08 \pm 0.001$ \\
\hline & Ind. and Inoc & $0.16 \pm 0.010$ & $0.24 \pm 0.011$ & $0.28 \pm 0.013$ & $0.09 \pm 0.002$ & $0.12 \pm 0.001$ & $0.16 \pm 0.012$ & $0.07 \pm 0.002$ & $0.14 \pm 0.010$ & $0.16 \pm 0.010$ \\
\hline \multirow[t]{2}{*}{$14 d$} & Ind. and Un-Inoc. & $0.14 \pm 0.001$ & $0.18 \pm 0.006$ & $0.21 \pm 0.010$ & $0.09 \pm 0.001$ & $0.10 \pm 0.006$ & $0.14 \pm 0.006$ & $0.07 \pm 0.001$ & $0.11 \pm 0.004$ & $0.15 \pm 0.006$ \\
\hline & Ind. and & $0.22 \pm 0.003$ & $0.30 \pm 0.005$ & $0.34 \pm 0.014$ & $0.11 \pm 0.001$ & $0.14 \pm 0.001$ & $0.19 \pm 0.013$ & $0.07 \pm 0.002$ & $0.18 \pm 0.011$ & $0.18 \pm 0.011$ \\
\hline \multicolumn{2}{|c|}{ A. sativum } & $5 \%$ & $10 \%$ & $15 \%$ & $5 \%$ & $10 \%$ & $15 \%$ & $5 \%$ & $10 \%$ & $15 \%$ \\
\hline \multirow[t]{2}{*}{$7 d$} & Ind. and Un-Inoc. & $0.06 \pm 0.003$ & $0.09 \pm 0.002$ & $0.12 \pm 0.004$ & $0.05 \pm 0.001$ & $0.07 \pm 0.004$ & $0.09 \pm 0.006$ & $0.02 \pm 0.001$ & $0.04 \pm 0.002$ & $0.06 \pm 0.002$ \\
\hline & Ind. and Inoc & $0.14 \pm 0.009$ & $0.19 \pm 0.013$ & $0.25 \pm 0.014$ & $0.08 \pm 0.010$ & $0.10 \pm 0.009$ & $0.15 \pm 0.013$ & $0.05 \pm 0.002$ & $0.08 \pm 0.003$ & $0.16 \pm 0.011$ \\
\hline \multirow[t]{2}{*}{$14 \mathrm{~d}$} & Ind. and Un-Inoc. & $0.17 \pm 0.012$ & $0.20 \pm 0.007$ & $0.25 \pm 0.011$ & $0.07 \pm 0.003$ & $0.08 \pm 0.011$ & $0.10 \pm 0.006$ & $0.03 \pm 0.006$ & $0.05 \pm 0.001$ & $0.07 \pm 0.003$ \\
\hline & Ind. and Inoc & $0.17 \pm 0.011$ & $0.22 \pm 0.013$ & $0.28 \pm 0.016$ & $0.08 \pm 0.002$ & $0.13 \pm 0.014$ & $0.17 \pm 0.011$ & $0.06 \pm 0.003$ & $0.10 \pm 0.010$ & $0.19 \pm 0.009$ \\
\hline
\end{tabular}

$H S D$ value for treatments $=0.0346 ;$ HSD value for doses $=0.00108 ;$ HSD value for varieties $=0.01454$.

Contrary to these results, increased nitrogen made plants more susceptible to pathogens in different hostpathogen interactions (Nam et al., 2006). Increasing nitrogen also increases lesion density with leaf rust (Robert et al., 2005). There are many other reports of increased severity of diseases with an increase in the concentration of leaf nitrogen (Olesen et al., 2003; Neumann et al., 2004).

The orthophosphate ion $\left(\mathrm{PO}_{4}^{3-}\right)$ has been documented by Walters and Bingham (2007) very significant for all cells, as it is included in various important molecules such as ATP, nucleic acids, and phosphoproteins.
Perrenoud (1990) directed effect of phosphorus on pathogen survival, development and multiplication; it may alter plant metabolism to affect a pathogen's food supply, stomatal function and plant defense to influence a pathogen's establishment in the plant. Our results confirmed that phosphorus increased in both, treated inoculated chickpea plants and treated un-inoculated but it increased more after inoculation with $A$. rabiei. Increased phosphorus in treated inoculated plants indicates that there are an intact and consistent uptake and translocation of phosphorus since it is used to form different cell constituents like phospholipids, nucleic acids, the coenzymes NADP, 
Table 3: Mean comparison ( \pm S.E) of potassium content ( $p p m$ ) in cultivars $C-44, P b-91$ and Bittle-98 after 7 and 14 days of the treatments with resistance inducers (chemicals and plant extracts).

\begin{tabular}{|c|c|c|c|c|c|c|c|c|c|}
\hline \multirow[t]{3}{*}{ Treatments } & \multicolumn{9}{|l|}{ Cultivars } \\
\hline & \multicolumn{3}{|l|}{$\mathrm{C}-44$} & \multicolumn{3}{|l|}{$\mathrm{Pb}-91$} & \multicolumn{3}{|l|}{ Bittle-98 } \\
\hline & \multicolumn{9}{|l|}{ Doses $(\mathrm{Mm})$} \\
\hline Salicylic acid & 0.5 & 1.0 & 1.5 & 0.5 & 1.0 & 1.5 & 0.5 & 1.0 & 1.5 \\
\hline 7d Ind. and Un-Inoc. & $167.85 \pm 1.19$ & $184.11 \pm 2.11$ & $200.96 \pm 0.98$ & $131.91 \pm 1.23$ & $145.44 \pm 2.34$ & $164.44 \pm 3.25$ & $68.91 \pm 1.46$ & $85.00 \pm 2.46$ & $94.93 \pm 2.52$ \\
\hline Ind. and Inoc & $480.86 \pm 6.71$ & $498.10 \pm 0.78$ & $509.57 \pm 4.92$ & $262.86 \pm 1.63$ & $283.05 \pm 2$ & $296.73 \pm 2.19$ & $120.13 \pm 2.05$ & $134.41 \pm 1.10$ & $146.22 \pm 1.15$ \\
\hline \multirow{2}{*}{$\begin{array}{l}\text { 14d Ind. and Un-In } \\
\text { Ind. and Inoc }\end{array}$} & $182.67 \pm 2.38$ & $195.96 \pm 2.93$ & $213.58 \pm 2.44$ & $141.01 \pm 3.38$ & $154.34 \pm 2.54$ & $164.79 \pm 2.96$ & $79.56 \pm 0.98$ & $92.26 \pm 1.49$ & $102.00 \pm 2.82$ \\
\hline & $491.88 \pm 4.87$ & $517.93 \pm 4.86$ & $527.86 \pm 6.64$ & $268.10 \pm 0.92$ & $288.89 \pm 4.97$ & $310.14 \pm 5.09$ & $131.16 \pm 1.08$ & $144.48 \pm 2.10$ & $158.32 \pm 1.51$ \\
\hline Bion & 0.4 & 0.8 & 1.2 & 0.4 & 0.8 & 1.2 & 0.4 & 0.8 & 1.2 \\
\hline 7d Ind. and Un-Inoc. & $182.63 \pm 1.89$ & $190.96 \pm 3.20$ & $204.37 \pm 2.63$ & $141.59 \pm 0.71$ & $154.47 \pm 2.81$ & $168.31 \pm 0.54$ & $71.59 \pm 0.89$ & $92.37 \pm 0.67$ & $103.62 \pm 0.71$ \\
\hline Ind. and Inoc & $491.81 \pm 1.00$ & $518.06 \pm 0.79$ & $528.43 \pm 5.29$ & $270.72 \pm 5.12$ & $288.91 \pm 5$ & $310.19 \pm 4.74$ & $131.94 \pm 0.94$ & $144.40 \pm 2.60$ & $158.03 \pm 1.10$ \\
\hline 14d Ind. and Un-Inoc. & $189.24 \pm 0$. & 195 & 207 & $144.66 \pm 0.53$ & 15 & $172.97 \pm 0.80$ & $76.97 \pm 0.71$ & 0.51 & 0.85 \\
\hline Ind. and Inoc & $495.68 \pm 2.79$ & $523.94 \pm 6.32$ & $533.01 \pm 4.90$ & $280.25 \pm 5.41$ & $297.63 \pm 5.20$ & $319.12 \pm 3.22$ & $139.85 \pm 2.52$ & $5 \pm 0.67$ & 7163. \\
\hline $\mathrm{KOH}$ & 25 & 50 & 75 & 25 & 50 & 75 & 25 & 50 & 75 \\
\hline 7d Ind. and Un-In & $83.93 \pm 1.60$ & $92.06 \pm 1.05$ & $100.49 \pm 1.52$ & $65.96 \pm 1.57$ & $72.73 \pm 1.62$ & $82.22 \pm 1.46$ & $34.46 \pm 0.50$ & $42.50 \pm 1.62$ & $47.47 \pm 0.64$ \\
\hline Ind. and Inoc & $240.43 \pm 6.11$ & $249.05 \pm 5.67$ & $254.79 \pm 3.37$ & $131.44 \pm 5.40$ & $141.53 \pm 0.41$ & $148.37 \pm 0.77$ & $60.07 \pm 1.58$ & $67.21 \pm 1.60$ & $73.11 \pm 0.57$ \\
\hline 14d Ind. and Un-Inc & $91.34 \pm 0.59$ & $97.98 \pm 1.01$ & $106.79 \pm 3.19$ & $70.51 \pm 0.48$ & $77.18 \pm 0.36$ & $82.40 \pm 1.48$ & $39.78 \pm 1.53$ & $46.13 \pm 1.49$ & $51.01 \pm 0.50$ \\
\hline Ind. an & $245.94 \pm 3.26$ & $258.97 \pm 3.92$ & $263.93 \pm 2.01$ & $134.06 \pm 1.94$ & $144.45 \pm 0.47$ & $155.07 \pm 2.73$ & $65.58 \pm$ & $72.24 \pm 0.28$ & $79.16 \pm 1.04$ \\
\hline A. indica & $5 \%$ & $10 \%$ & $15 \%$ & $5 \%$ & $10 \%$ & $15 \%$ & $5 \%$ & $10 \%$ & $15 \%$ \\
\hline 7d Ind. and Un-Inoc. & $54.55 \pm 0.73$ & $59.83 \pm 0.80$ & $65.31 \pm 0.97$ & $42.87 \pm 1.32$ & $47.27 \pm 0.77$ & $49.82 \pm 0.30$ & $22.40 \pm 0.33$ & $27.63 \pm 0.27$ & $30.85 \pm 0.41$ \\
\hline Ind. and Inoc & $156.28 \pm 1.96$ & $162.55 \pm 1.51$ & $165.61 \pm 2.24$ & $85.77 \pm 1.04$ & $92.33 \pm 0.88$ & $96.77 \pm 1.52$ & $39.05 \pm 0.32$ & $43.69 \pm 0.35$ & $47.52 \pm 0.77$ \\
\hline 14d Ind. and Un-Inoc. & $59.37 \pm 0.89$ & $63.69 \pm 1.57$ & $69.41 \pm 0.36$ & $45.83 \pm 0.31$ & $50.17 \pm 0.24$ & $53.56 \pm 1.56$ & $25.86 \pm 0.32$ & $29.99 \pm 0.71$ & $33.16 \pm 0.32$ \\
\hline Ind. and Inoc & $159.86 \pm 5.44$ & $168.66 \pm 1.06$ & $174.89 \pm 2.23$ & $87.14 \pm 0.87$ & $96.56 \pm 0.49$ & $103.46 \pm 2.02$ & $42.63 \pm 0.92$ & $47.62 \pm 0.70$ & $52.12 \pm 0.92$ \\
\hline D. metel & $5 \%$ & $10 \%$ & $15 \%$ & $5 \%$ & $10 \%$ & $15 \%$ & $5 \%$ & $10 \%$ & $15 \%$ \\
\hline $7 \mathrm{~d}$ Ind. and $\mathrm{Un}-\mathrm{Ir}_{\mathrm{r}}$ & $35.10 \pm 0.19$ & $38.83 \pm 0.50$ & $42.35 \pm 0.50$ & $26.58 \pm 0.76$ & $29.75 \pm 0.86$ & $34.38 \pm 0.28$ & $14.08 \pm 0.50$ & $17.44 \pm 0.35$ & $19.85 \pm 0.27$ \\
\hline Ind. and Inoc & $101.55 \pm 0.73$ & $104.48 \pm 2.56$ & $109.22 \pm 0.74$ & $55.63 \pm 0.68$ & $59.85 \pm 0.80$ & $64.71 \pm 2.09$ & $25.45 \pm 0.48$ & $28.44 \pm 0.55$ & $31.24 \pm 0.65$ \\
\hline 14d Ind. and Un-Ino & $38.53 \pm 0.52$ & $42.97 \pm 1.33$ & $45.99 \pm 0.69$ & $30.15 \pm 0.85$ & $33.94 \pm 0.78$ & $35.46 \pm 0.63$ & $16.63 \pm 0.20$ & $19.63 \pm 0.22$ & $21.66 \pm 0.36$ \\
\hline Ind. and Inoc & $104.18 \pm 2.84$ & $108.60 \pm 1.65$ & $116.37 \pm 1.69$ & $58.06 \pm 1.53$ & $66.41 \pm 1.53$ & $69.18 \pm 0.90$ & $29.09 \pm 1.03$ & $31.21 \pm 0.69$ & $36.10 \pm 0.77$ \\
\hline A. sativum & $5 \%$ & $10 \%$ & $15 \%$ & $5 \%$ & $10 \%$ & $15 \%$ & $5 \% \pm$ & $10 \%$ & $15 \%$ \\
\hline 7d Ind. and Un-Inoc. & $27.14 \pm 0.14$ & $30.10 \pm 0.46$ & $32.82 \pm 0.46$ & $20.99 \pm 0.98$ & $23.85 \pm 0.79$ & $26.92 \pm 0.71$ & $11.14 \pm 0.16$ & $13.74 \pm 0.13$ & $15.35 \pm 0.20$ \\
\hline Ind. and Inoc & $78.07 \pm 1.02$ & $81.53 \pm 1.63$ & $83.38 \pm 2.01$ & $43.17 \pm 1.64$ & $48.43 \pm 1.55$ & $51.64 \pm 1.32$ & $20.09 \pm 0.77$ & $23.73 \pm 1.14$ & $25.97 \pm 1.36$ \\
\hline 14d Ind. and Un-Inoc. & $29.86 \pm 1.05$ & $33.35 \pm 1.02$ & $35.53 \pm 1.16$ & $22.80 \pm 0.65$ & $25.29 \pm 0.81$ & $28.31 \pm 0.95$ & $12.86 \pm 0.16$ & $15.92 \pm 0.90$ & $17.49 \pm 1.15$ \\
\hline Ind. and Inoc & $79.86 \pm 1.60$ & $86.73 \pm 2.57$ & $89.34 \pm 1.65$ & $43.68 \pm 1.29$ & $49.70 \pm 1.83$ & $59.47 \pm 1.82$ & $21.21 \pm 0.17$ & $25.36 \pm 1.08$ & $29.26 \pm 0.78$ \\
\hline
\end{tabular}

$H S D$ value for treatments $=19.7488 ;$ HSD value for doses $=0.4058 ;$ HSD value for varieties $=3.0674$.

NAD ATP, and other energy-yielding compounds. Almost similar findings were reported by Sahi et al. (2007) who reported that Phosphorus was higher in un-inoculated plants of susceptible lentil lines than the resistant ones, but upon inoculation Phosphorus also increased in resistant lentils. The present results are also consistent with Randhawa (1994) who reported an increase in phosphorus contents in chickpea cultivars of both resistant and susceptible groups after infection with Ascochyta rabiei. The application of foliar phosphorus induced systemic protection (PISP) against powdery mildew of roses, mango, and nectarines (Reuveni and Reuveni, 1998) by increasing the activity of peroxidase, phenylalanine, lipoxygenase, and ammonia-lyase (PAL). A similar effect might occur in this case where increased phosphorus $(\mathrm{P})$ induced systemic resistance in chickpea plants. Increased phosphorus reduces the intensity of disease in different host-pathogen interactions with downy mildew, rice bacterial blight, blue mold, barley yellow dwarf virus and rice blast (Huber and Graham, 1999; Reuveni et al., 2000). Phosphate applied to plants might direct apoplastic calcium to amend membrane integrity and therefore influence the movement of apoplastic enzymes and discharge elicitor-active oligogalacturonides from the cell wall of different 
plants (Gottstein and Kuc, 1989; Walters and Murray, 1992). On the other hand, phosphorus may enhance the severity of sclerotinia in garden plants and downy mildew of onion (Develash and Sugha, 1997). The results of our research is consistent with that of Shehu and Aliero (2010) who reported increased phosphorus in leaves inoculated with Alternaria porri compared to healthy ones.

Application of the proper rate of potassium, balanced with other nutrients, decreases the susceptibility of plants (Huber and Graham, 1999). Potassium is involved in different metabolic functions (respiration, photosynthesis) and the synthesis of high molecular weight compounds. Potassium deficiency increases parasitic diseases due to a disturbance of metabolic functions. Present studies indicated that there is increased potassium in chickpea cultivars especially cultivar C-44, after chemical treatment and inoculation with $A$. rabiei. This noticeable increase in potassium of chemically treated; inoculated susceptible chickpea may be attributed to a change in the physiological and biochemical plant defense system. Similarly, Sahi et al. (2007) found that potassium increased in both resistant and susceptible lentil lines after inoculation with $A$ lentis. These results are also consistent with those of Randhawa (1994) and Reddy and Khare (1984). Our results are consistent with El-Khallal (2007) who reported high potassium in salicylic acid-treated plants. A converse association between disease incidence and potassium nutrition has been revealed for sheath blight of rice (Thanatephorus cucumeris), rice blast (Pyricularia oryzae). A restorative effect of potassium was reported for bacterial diseases in rice such as bacterial leaf blight (Krauss, 1999). By increasing potassium availability and uptake the severity of stripe rust decreased. Almost 2450 references were reviewed by Perrenoud (1990) on the use of potassium. He reported that $70 \%$ of fungal diseases were decreased, 69\% of bacterial diseases, 63 $\%$ of mites and insects and $41 \%$ of virus diseases by potassium. Higher applications of potassium increased the yield of fungal, bacterial, and viral pathogen-infected plants. Potassium deficiency results in decreased of protein synthesis and accumulation of simple nitrogen compounds such as amides that are used by plant pathogens (Dordas, 2008). There were no observed differences in crop response concerning different potassium sources. The balance between nitrogen and potassium affects the disease susceptibility of plants. Examples of disease reduction in obligate and facultative parasites by potassium included severity of leaf blight (Helminthosporium) and increase wheat yield and seedling rot caused by Rbizoctonia solani (Sharma and Duveiller 2004; Sharma et al., 2005). Excess potassium harmed the growth of tomato plants because they were more susceptible to Verticillium wilt caused by $V$. dahliae (Burge and Simmons, 1982).

\section{Acknowledgments}

The authors are highly grateful to Dr. Shakeel Ahmad (Head of Research and Development, Syngenta), Pakistan for providing Bion and all support for its import from Germany.

\section{Novelty Statement}

Adequate nutrition plays a key role in mitigating disease damage. Hence, current study indicates that chemicals reported to induce resistance to plant pathogens may increase mineral contents which prevent the spread of the pathogen.

\section{Author's Contribution}

Dr. Muhammad Usman Ghazanfar conceived and designed research and wrote the manuscript. Dr. Waqas Wakil analyzed the data and help in conduct of experiments. Dr. Shahbaz Talib Sahi supervised the experiment and improved the draft. Dr. Waqas Raza helped in analysis, submission and correspondence of paper.

\section{Conflict of interest}

The authors have declared no conflict of interest.

\section{References}

Agrios, G.N., 2005. Plant pathology. $5^{\text {th }}$ Ed. Elsevier-Academic Press USA.

Ahmad, H.U., K.F. Chang, S.F. Hwang, B.D. Gossen, R.J. Haward and T.D. Warkintin. 2006. Components of disease resistance in Desi and Kabuli chickpea varieties against Ascochyta blight. Plant Pathol. J., 5: 336-342. https://doi. org/10.3923/ppj.2006.336.342

Ajay, D. and U.I. Baby. 2009. Induction of systemic resistance to Exobasidium vexans in tea through SAR elicitors. Phytopara, 38: 53-60. https:// doi.org/10.1007/s12600-009-0068-x

Amtmann, A., S. Troufflard and P. Armengaud. 
2009. The effect of potassium nutrition on pest and disease resistance in plants. Physiol. Plant, 133: 682-691. https://doi.org/10.1111/j.13993054.2008.01075.x

Bhargava,B.S. and H.B.Raghupathi.1995. Analysis of plant material for macro and micronutrients. In: Tandon H.L.S. (ed.). Methods of analysis of soils, plants, waters and fertilizers, Fertilizer Development and Consultation Organization, New Delhi, India. pp. 49-82.

Borges, A.A., A. Doben, M. Expósito-Rodríguez, D. Expósito-Rodríguez, A. Borges-Pérez, V. Casañas-Sánchez, J.A. Pérez, J.C. Luis, P. Tornero. 2009. Molecular analysis of menadione-induced resistance against biotic stress in Arabidopsis. Plant Biotech J., 7: 744-762. https://doi.org/10.1111/j.14677652.2009.00439.x

Burge, M.N.,J.R. Simmons. 1982. The influence of potassium and phosphorus in predisposition of tomato toVerticilliumwilt.Chem.Ecol.,1:83-92. https://doi.org/10.1080/02757548208070790

Chandra, D. and P. Mishra. 1991. Effect of different level of nitrogen, phosphorus, potassium application on grain and milling yield of "Gayarti" rice (Oryza sativa L.). Indian J. Agric. Sci., 61: 496-498.

Chen, Y.M. and R.N. Strange. 1991. Synthesis of the solanapyrone phytotoxin by Ascochyta rabiei in response to metal cations and development of a defined medium for toxin production. Plant Pathol., 40: 401-407. https://doi. org/10.1111/j.1365-3059.1991.tb02397.x

Develash, R.K. and S.K. Sugha. 1997. Factors affecting development of downy mildew (Peronospora destructor) of onion (Allium cepa). Indian J. Agric. Sci., 67: 71-74. https://doi. org/10.1016/S0261-2194(96)00056-7

Dietrich,R.,K.Plob and M.Heil.2004.Constitutive and induced resistance to pathogens in Arabidopsis thaliana depends on nitrogen supply. Plant Cell Environ., 27: 896-906. https://doi. org/10.1111/j.1365-3040.2004.01195.x

Dordas, C., 2008. Role of nutrients in controlling plant diseases in sustainable agriculture. A Review. Agron. Sustain.Dev.,28:33-46.https:// doi.org/10.1051/agro:2007051

El-Khallal, S.M., 2007. Induction and modulation of resistance in tomato plants against Fusarium wilt disease by bioagent fungi (arbuscular mycorrhiza) and/or hormonal elicitors
(Jasmonic acid and salicylic acid): 1-changes in growth, some metabolic activities and endogenous hormones related to defence mechanism. Aust. J. Basic Appl. Sci., 1: 691705.

Filippi, M.C. and A.S. Prabhu. 1998. Relationship between panicle blast severity and mineral nutrient content of plant tissue in upland rice. J. Plant Nutr., 21: 1577-1587. https://doi. org/10.1080/01904169809365505

Fuchs, W.H. and F. Grossmann. 1972. Ernährung und resistenz von kulturpflanzen gegenüber krankheitserregern und schädlingen [Nutrition and resistance of crop plants against pathogens and pests]. In: Linser, H., (ed.). Handbuch der pflanzenernährung und duüngung. SpringerVerlag, Vienna. 1(2): 1008-1107.

Ghazanfar, M.U., S.T. Sahi, N. Javid and W. Wakil. 2010. Response of advanced lines of chickpea against chickpea blight disease. Pak. J. Bot., 42: 3423-3430.

Gottstein, H.D. and J. Kuc. 1989. Induction of systemic resistance to anthracnose in cucumber by phosphates. Phytopathology, 79: 176-179. https://doi.org/10.1094/Phyto-79-176

Graham,D.R. and M.J.Webb.1991.Micronutrients and disease resistance and tolerance in plants, In: Mortvedt,J.J., Cox, F.R., Shuman, L.M., Welch, R.M., (ed.). Micronutrients in Agriculture, $2^{\text {nd }}$ ed. Soil Sci. Society of America, Inc. Madison, Wisconsin, USA. pp. 329-370. https://doi. org/10.2136/sssabookser4.2ed.c10

Guleria, S. and A. Kumar. 2006. Azadirachta indica leaf extract induces resistance in sesame against Alternaria leaf spot disease. J. Cell Mol. Biol., 5: 81-86.

Hoffland, E., M.J. Jegger, L.M. Beusichem. 2000. Effect of nitrogen supply rate on disease resistance in tomato depends on the pathogen. Plant Soil., 218: 239-247. https://doi. org/10.1023/A:1014960507981

Huber, D.M. and R.D. Graham. 1999. The role of nutrition in crop resistance and tolerance to disease. In: Rengel, Z., (ed.). Mineral nutrition of crops fundamental mechanisms and implications, Food Product Press, New York. pp. 205-226.

Ilyas, M.B. and I.U. Khan. 1986. A low cost easy technique for the culturing of Ascochyta rabiei fungus. Pak. J. Agric. Sci., 23: 60-66.

Jaganathan, R. and V. Narasimhan. 1988. Effect of 
plant extracts products on two fungal pathogens of finger millet. Indian J. Mycol. P1. Pathol., 18: 250-254.

Kagale, S.T., T. Marimuthu, P. Thaynmanavan, P. Nandakumar and R. Samiyappan. 2004. Antimicrobial activity and induction of systemic resistance in rice by leaf extract of Dathura metel against Rhizoctonia solani and Xanthomonas oryzae pv. oryzae. Physiol. Mol. P1. Pathol., 65: 91-100. https://doi.org/10.1016/j. pmpp.2004.11.008

Kalara, Y.P. and D.G. Maynard. 1991. Methods manual for forest soil and plant analysis. For. Can., Northwest Reg., North. For. Cent., Edmonton, Alberta. Inf. Rep. NOR-X-319.

Kiraly, Z., 1976. Plant disease resistance as influenced by biochemical effects of nutrients and fertilizers. In: Fertilizer use and plant health, proceedings of colloquium 12. Atlanta, GA: International Potash Institute, pp. 33-46.

Kone, D., A.S. Csinos, K.L. Jackson and P.Ji. 2009. Evaluation of systemic acquired resistance inducers for control of Phytophthora capsici on squash. Crop Prot., 28: 533-538. https://doi. org/10.1016/j.cropro.2009.02.005

Krauss, A., 1999. Balanced nutrition and biotic stress. In: IFA agricultural conference on managing plant nutrition held at Barcelona, Spain, from 29 ${ }^{\text {th }}$ June- 2 July 1999.

Kuc, J., 2001. Concepts and direction of induced systemic resistance in plants and its application. Eur. J. Plant Pathol., 107: 7-12.

Luthra,J.C., A. Sattar, K.S. Bedi. 1935. Life history of gram blight Ascochyta rabiei (Pass.) Labr. and control measures for its control. Proc. Indian Sci. Conf., 22: 374-374.

Malik, B.A. and M. Bashir. 1984. Strategies for controlling gram blight. Prog. Farm., 4: 21-23.

Marschner, H., 1995. Mineral nutrition of higher plants. $2^{\text {nd }}$ Ed. Academic Press, London.

Nam, M.H., S.K. Jeong, Y.S. Lee, J.M. Choi and H.G. Kim. 2006. Effects of nitrogen, phosphorus, potassium and calcium nutrition on strawberry anthracnose. Plant Pathol., 55: 246-249. https://doi.org/10.1111/j.13653059.2006.01322.x

Nene, Y.L. and M.V. Reddy. 1987. Chickpea diseases and their control. In: Saxena M.C., Singh K.B. (Ed.). The Chickpea. CAB International, ICARDA, Syria. pp. 233-270.

Nene, Y.L., 1984. A review of Ascochyta blight of chickpea. In: Saxena, M.C., Singh, K.B. (ed.). Ascochyta blight and winter sowing of chickpea. Martinus Nighoff/ Dr. W. Junk Publishers. The Hague, Netherland. pp.17-36.

Neumann, S., N.D. Paveley, F.D. Beed and R. Sylvester-Bradley. 2004. Nitrogen per unit area affects the upper asymptote of Puccinia striiformis f.sp. tritici epidemics in winter wheat. Plant Pathol., 53: 725-732. https://doi. org/10.1111/j.1365-3059.2004.01107.x

Olesen, J.E., L.N. Jorgensen, J. Petersen and J.V. Mortensen. 2003. Effects of rates and timing of nitrogen fertilizer on disease control by fungicides in winter wheat. 2. Crop growth and disease development. J. Agric. Sci., 140: 15-29. https://doi.org/10.1017/S0021859602002897

Oostendorp, M., W. Kunz, B. Dietrich and T. Staub. 2001. Induced resistance in plants by chemicals. Eur. J. P1. Pathol., 107: 19-28. https://doi. org/10.1023/A:1008760518772

Peoples, T.R. and D.W. Koch. 1979. Role of potassium in carbon dioxide assimilation in Medicago sativa L. Plant Physiol., 63: 878-881. https://doi.org/10.1104/pp.63.5.878

Perrenoud, S., 1990. Potassium and plant health. IPI-Research Topics No. 3, 2"d Rev. edition. International Potash Institute, Basel, Switzerland. pp. 365.

Randhawa,M.A.,1994.Role of some morphological and chemical characters of gram in resistance to Ascochyta blight. Ph.D. thesis, University of Agriculture, Faisalabad, Pakistan.

Reddy, R.R. and M.N. Khare. 1984. Further studies on factors influencing the mechanism of resistance in lentil (Lens culinaris M.) to rust (Uromyces fabae (Pers.) de Bary. Lens Newsl., $11: 29-32$.

Reuveni, R. and M. Reuveni. 1998. Foliar fertilizer therapy a concept in integrated pest management. Crop Prot., 17: 111-118. https:// doi.org/10.1016/S0261-2194(97)00108-7

Reuveni, R., G. Dor, M. Raviv, M. Reuveni and S. Tuzun. 2000. Systemic resistance against Sphaerotheca fuliginea in cucumber plants exposed to phosphate in hydroponics system and its control by foliar spray of mono-potassium phosphate. Crop Prot., 19: 355-361. https:// doi.org/10.1016/S0261-2194(00)00029-6

Robert, C., M.O. Bancal, B. Ney and C. Lannou. 2005. Wheat leaf photosynthesis loss due to leaf rust, with respect to lesion development 
and leaf nitrogen status. New Phytol., 165: 227-241. https://doi.org/10.1111/j.14698137.2004.01237.x

Ruan, J. and X. Wu. 2000. Balanced plant nutrition may help reduce pesticide use by improving tea plants resistance to fungal diseases. UNEP Industry and Environment. January-June. pp. 89-90.

Sahi, S.T., M.U. Ghazanfar, M.Afzal, A. Habib and M.B. Ilyas. 2007. Role of N, P and K contents in resistance against Ascochyta blight of lentil (Lens Culinaris Medik). Pak J. Bot., 39: 21752181.

Sattar, A., 1933. On the occurrence, perpetuation and control of gram blight caused by Ascochyta rabiei with special reference to Indian conditions. Ann. Appl. Biol., 20: 612-632. https://doi. org/10.1111/j.1744-7348.1933.tb07448.x

Sharma, R.C. and E. Duveiller. 2004. Effect of Helminthosporium leaf blight on the performance of timely and late-seeded wheat under optimal and stressed levels of soil fertility and moisture. Field Crop Res., 89: 205-218. https://doi.org/10.1016/j.fcr.2004.02.002

Sharma, S., E. Duveiller, R. Basnet, C.B. Karki and R.C.Sharma.2005. Effect of potash fertilization on Helminthosporium leaf blight severity in wheat and associated increases in grain yield and kernel weight. Field Crop Res., 93: 142150. https://doi.org/10.1016/j.fcr.2004.09.016

Shehu, K. and A.A. Aliero. 2010. Effects of purple blotch infection on the proximate and mineral contents of onion leaf. Int. J. Pharm. Sci. Res., 1: 131-133.

Singh, G. and M. Singh. 1990. Chemical control of Ascochyta blight of chickpea. Indian Phytopathol., 43: 59-63.

Slusarenko, J.A., A. Patel, D. Portz. 2008. Control of plant diseases by natural products: Allicin from garlic as a case study. European J. P1.
Pathol., 121: 313-322. https://doi.org/10.1007/ s10658-007-9232-7

Solomon, P.S. and R.P. Oliver. 2001. The nitrogen content of tomato leaf apoplast increases during infection by Gladosporium flavum. Planta, 213: 241-249. https://doi.org/10.1007/ s004250000500

Solomon, P.S., K. Tan and R.P. Oliver. 2003. The nutrient supply of pathogenic fungi; a fertile field for study. Mol. Plant Pathol., 4: 203-210. https://doi.org/10.1046/j.13643703.2003.00161.x

Steel, R.G.O., J.H. Torrie and D. Dickey. 1997. Principles and procedures of statistics. A biometrical approach. $3^{\text {rd }}$ ed. Mc Graw-Hill, New York. U.S.A.

Stitt,M.and A.Krapp.1998.The interaction between elevated carbon dioxide and nitrogen nutrition: The physiological and molecular background. Plant Cell Environ., 22: 583-621. https://doi. org/10.1046/j.1365-3040.1999.00386.x

Tenhaken, R., and W. Barz. 1991. Characteristics of peptic enzymes from the chickpea pathogen Ascochyta rabiei. Z. Naturforsch., 46: 51-57. https://doi.org/10.1515/znc-1991-1-209

Tripathi, H.S., R.S. Singh and H.S. Chanbe. 1987. Effect of fungicidal seed and foliar application on chickpea Ascochyta blight. Indian Phytopathol., 40: 63-66.

Walters, D.R. and D.C. Murray. 1992. Induction of systemic resistance to rust in Vicia faba by phosphate and EDTA: Effects of calcium. Plant Pathol., 41: 444-448. https://doi. org/10.1111/j.1365-3059.1992.tb02439.x

Walters, D.R. and I.J. Bingham. 2007. Influence of nutrition on disease development caused by fungal pathogens: implications for plant disease control.Ann. Appl. Biol., 151:307-324. https:// doi.org/10.1111/j.1744-7348.2007.00176.x 